\title{
Processes After Cold Exposure Leading to the Onset of Stroke
}

\author{
Hiroshi Morimoto*
}

Department of Mathematical Modeling and Informatics Unit, Graduate School of Informatics, Nagoya University, Japan

*Corresponding author: Hiroshi Morimoto, Department of Mathematical Modeling and Informatics Unit, Graduate School of Informatics, Nagoya University, Japan, Tel: +81 052789 4716; E-mail: hiroshim@info.human.nagoya-u.ac.jp

Received date: November 06, 2017; Accepted date: November 24, 2017; Published date: November 30, 2017

Copyright: (C) 2017 Morimoto $\mathrm{H}$. This is an open-access article distributed under the terms of the Creative Commons Attribution License, which permits unrestricted use, distribution, and reproduction in any medium, provided the original author and source are credited.

Citation: Morimoto H (2017) Processes After Cold Exposure Leading to the Onset of Stroke. Eur Exp Biol Vol. 7 No. 6:41.

\section{Abstract}

Background: The mortality from stroke is known to increase to a greater extent with the fall of temperature. Environmental factors may play a major role in triggering of the onset of stroke. There is a delay between cold exposure and the onset of stroke. This fact may reflect the complex mechanism after cold exposure. However, a precise process after cold exposure is not well documented. The purpose of this paper is to study the processes after cold exposure that affect human health, exploring the changes in both weather and gene expression.

Methods and findings: We used ambulance data of patients provided by the city of Nagoya in Japan during a period from 2002 to 2005. The data included the daily number of patients of all ages, who were first transported by ambulance to a hospital and then diagnosed at the hospital with stroke or cerebral infarction. We used also gene expression data extracted from the database GEO (gene expression omnibus) maintained by The National Center for Biotechnology Information (NCBI). The method is based on random walks in the plane of two variables, mean temperature and relative humidity. We extracted random walks that correspond to paths after cold exposure leading to the onset of stroke and classified the characteristic feature of cooling and rewarming random walks. The increase of relative humidity was observed during these random walks, and suggested the importance of inflammatory system. To discuss a possible mechanism after cold exposure, we examined the gene expression data among the database GEO, and found the increase of cytokine-related genes, especially interleukin 6 signal transducer IL6st and interleukin receptors of IL17. These interleukins play an important role in inflammatory responses.

Conclusion: The correspondence between the gene expressions of cytokines and "cooling and rewarming random walks" in the meteorological factors will provide a scheme that connects weather and disease, and will provide novel avenues for research in the prevention of stroke.

Keywords: Stroke; Gene expression; Cytokine; Interleukin; Random walk

\section{Introduction}

The increase of mortality from stroke was observed with the fall of temperature [1]. It is hypothesized that exposure to winter weather conditions may induce the increase of the incidence or case fatality of stroke [2]. These studies suggested that environmental factors might play a major role in the triggering of stroke events or in determining their outcome. An understanding of the potential role of seasonal stresses and mechanisms responsible for these effects may issue an innovative approach for research in the prevention of stroke.

One of the characteristic features of diseases affected by cold exposure is the appearance of delay between the event of cold exposure and the onset of disease. To clarify the reason of these lags, it is important to understand the process happening after cold exposure from a viewpoint of both weather and disease. However, changes after cold exposure is not well documented. The purpose of this paper is to study the processes after cold exposure that affect human health, exploring the changes in both weather and gene expression.

A rewarming procedure observed between cold exposure and the onset of stroke was pointed out by association analysis $[3,4]$. This pattern of weather was called "cooling and rewarming". At the same time, the increase of relative humidity was also observed during this cooling and rewarming procedure. To understand these procedures, we adopted in this paper the method of random walk. If we consider the plane of two variables, mean temperature $T$ and relative humidity $H$, then at each day, $t$, the pair $T(t)$ and $H(t)$ can be plotted as a point in the plane $(T, H)$. As day, $t$, changes, the corresponding point will change the position in the plane $(T, H)$. This process will produce a random walk in the plane. Thus the daily variability of mean temperature and relative humidity can be understood as a random walk in the plane $(T, H)$.

Among these random walks, the random walks ending with high risk cases of stroke are interesting for the purpose of forecasting the onset of stroke. In particular, in a case of cooling and rewarming, the corresponding random walks were named "cooling and rewarming random walks". We explored these "cooling and rewarming random walks" ending with the onset of stroke. 
On the other hand, it is known that cold stress triggers a complex procedure of gene expression and metabolic responses $[5,6]$. The studies of these responses in gene levels are of importance to therapeutic manipulation. A scheme that connects weather and disease was proposed in terms of cytokine networks [7-9]. The network consists of interleukins, where IL-6 plays an important role. An investigation of gene expression including IL- 6 family and related genes will be of scientific importance. We investigated the change of gene expression of several interleukins that might be corresponding to cooling and rewarming random walks, using the gene expression data extracted from gene expression omnibus (GEO) maintained by the national center for biotechnology information (NCBI).

\section{Methods}

We used patient ambulance data provided by the city of Nagoya, which consisted of the daily data of the number of patients during a period from 2002 to 2005 . The data included the number of patients of all ages, who were first transported by ambulance to a hospital and then diagnosed at the hospital with stroke or cerebral infarction. Nagoya is located on central land of Japan and it has a moderate standard subtropical climate. We also used daily meteorological data such as the daily mean temperature and relative humidity, extracted from Japan Meteorological Agency.

As data of DNA, we used gene expression data extracted from the database, GEO (gene expression omnibus) which is maintained by The National Center for Biotechnology Information (NCBI).

NCBI is a database (called GenBank) of DNA sequences providing access to biomedical and genomic information. GEO is a public functional genomics data repository organized by NCBI and stores curated gene expression datasets. We used gene expression data with the accession numbers, GSE13432 and GSE70437. The data of accession number GSE13432 was originally supplied by Xue et al. [9]. The data were obtained from microarrays for the purpose of investigating molecular mechanisms of angiogenesis in relation to adipose tissue metabolism. They exposed adult mice to $4^{\circ} \mathrm{C}$ for up to 5 weeks. Control mice were maintained at their thermoneutral temperature of $30^{\circ} \mathrm{C}$. We used control data exposed to $30^{\circ} \mathrm{C}$ rather than those data exposed to cold $\left(4^{\circ} \mathrm{C}\right)$ to examine the effect of warming process. The data of accession number GSE70437 was uploaded to the database GEO by Marcher et al. [10]. The data contained the gene expression data of mice kept at either $4^{\circ} \mathrm{C}$ or $22^{\circ} \mathrm{C}$ for three days.

As a method, we used "random walks" [11,12]. The two variables, mean temperature and relative humidity, forms a two dimensional plane (denoted by $\mathrm{S}$ ). The pair of the mean temperature $T(t)$ for some day $t$ and the relative humidity $H(t)$ of the same day $t$ can be considered as a point $P(t)=(T(t), H(t))$ in the plane $S$. As time or day $t$ changes like $t, t+1, t+2, \ldots$, the corresponding point changes like $P(t), P(t+1), P(t+2), \ldots$, forming a sequence of points in the plane $S$. This process defines a random walk in the plane $S$,

$$
P(t)=(T(t), H(t)), P(t+1)=(T(t+1), H(t+1)), \ldots, P(n)=(T(n), H(n)), \ldots
$$

We explored these random walks that end at a high risk day of cerebral infarction and examined characteristic feature of these random walks.

The feature of relative humidity along random walks suggested the importance of inflammatory system and motivated us to examine gene expression of interleukins, especially IL-6 that plays an important role in inflammatory system.

\section{Results}

We classified all of the days into three classes, namely, 'high risk day', 'moderate risk day' and 'low risk day' according to the amount of patients with stroke. The high (or low) risk cases were defined to occupy $30 \%$ among the total cases during winter terms in 2003-2005.

Now suppose that $t$ is the day belonging to high risk days, i.e., the number of patients of the day $t$ scored a large number. $T(t)$ and $\mathrm{H}(\mathrm{t})$ denotes the mean temperature and the relative humidity of the day $t$, respectively. The pair $\mathrm{T}(\mathrm{t})$ and $\mathrm{H}(\mathrm{t})$ can be considered as a point $\mathrm{P}(\mathrm{t})=(\mathrm{T}(\mathrm{t}), \mathrm{H}(\mathrm{t}))$ in a two dimensional plane. The history, $(T(t-k), H(t-k)), \ldots$, before high risk day is important for the forecasting the incidence of the disease at day t.

We defined "random walk" as the history during one week for the given high risk day, as follows.

Consider the plane of two variables, mean temperature and relative humidity. A random walk is a sequence of points in this plane;

$P(t-6)=(T(t-6), H(t-6)), P(t-5)=(T(t-5), H(t-5)), \ldots, P(t-1)=(T(t-1)$, $H(t-1)), P(t)=(T(t), H(t))$
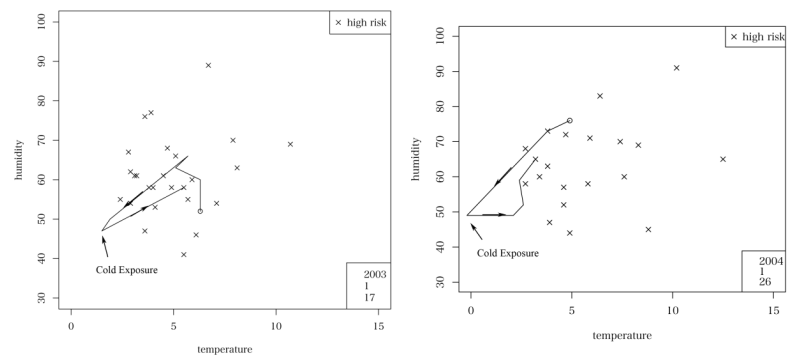

Figure 1: Cooling and rewarming random walks in winter seasons. Cross marks represent days of high risk in winter seasons in 2003 and 2004, respectively. These are the random walks that end on January 17 in 2003 (left) and January 26 in 2004 (right). The starting point is marked with circle and the end point with cross. The sharp edges on the left bottom correspond to cold exposure. The increase in relative humidity is observed in both cases.

Starting from $\mathrm{P}(\mathrm{t}-6)$ and reaching $\mathrm{P}(\mathrm{t})$ of the high risk day. An example can be seen in Figure 1, where the random walk starts 
with circle mark and ends with cross mark (high risk case). Cooling and rewarming cases can be interpreted as a random walk $\mathrm{P}(\mathrm{t}-6), \ldots, \mathrm{P}(\mathrm{t}-\mathrm{k}), \ldots, \mathrm{P}(\mathrm{t})$, where cold exposure occurred at some day, $t-k$ and then after that, a random walk $P(t-k+1), \ldots$, $\mathrm{P}(\mathrm{t})$ becomes a warming process, i.e., $\mathrm{H}(\mathrm{t}-\mathrm{k}+1), \ldots, \mathrm{H}(\mathrm{t})$ are generally increasing.

Since the above definition of cooling and rewarming appears intuitive, we give a more mathematical definition of "cooling and rewarming random walk", as follows. We first calculate the minimum of the temperatures $T(t-6), \ldots, T(t)$, and denote it by $T m$. Then, consider three values $T m, T(t-6), T(t)$, i.e., the minimum, the start, and the end. If $\mathrm{Tm}$ is relatively smaller than $T(t-6)$, then Tm can be considered as a cold exposure. More precisely, the condition of cold exposure may be written $\mathrm{Tm}<\mathrm{T}(\mathrm{t}-6)-2^{\circ} \mathrm{C}$ if we select $\left(2^{\circ} \mathrm{C}\right)$ as an appropriate difference to feel cold. If $T(t)$ is larger than $T m$, then the temperature increased from $T m$ to $T(t)$. Therefore if the following conditions are satisfied at the same time, then we define the random walk, $\mathrm{w}(\mathrm{t}-6), \ldots, \mathrm{w}(\mathrm{t})$ as a "cooling and rewarming walk";

$\operatorname{Tm}<\mathrm{T}(\mathrm{t}-6)-2^{\circ} \mathrm{C}$ and $\mathrm{Tm}<\mathrm{T}(\mathrm{t})-2^{\circ} \mathrm{C}$

As a result, the cooling and rewarming random walk has a following feature:

$\mathrm{T}(\mathrm{t}-6)[=$ start $] \rightarrow$ cooling $\rightarrow \mathrm{Tm}[=$ cold exposure $] \rightarrow$ rewarming $\rightarrow \mathrm{T}(\mathrm{t})[=\mathrm{end}]$

We explored the data during winter seasons from 2002 to 2005, and checked all the random walks ending with the day of high risk of cerebral infarction. Then we selected, from these random walks, all the cooling and rewarming random walks. Typical examples are shown in Figures $\mathbf{1}$ and $\mathbf{2}$.

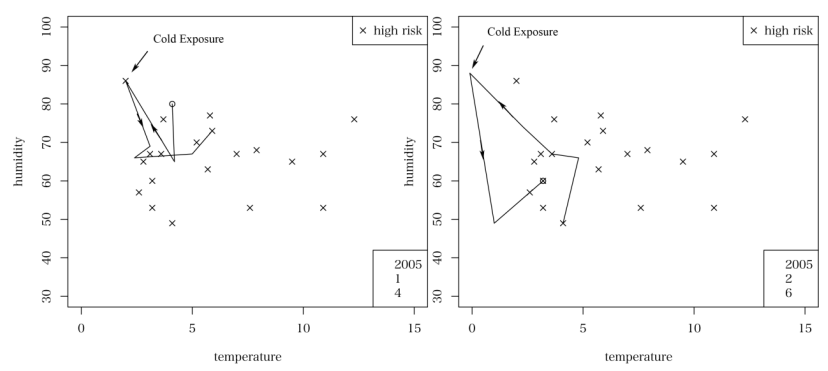

Figure 2: Cooling and rewarming random walks with high humidity at cold exposure. These are the random walks that end on January 4 in 2005 (left) and February 6 in 2005 (right). The starting point is marked with circle and the end point with cross. The sharp edges on the left upper corner correspond to cold exposure. The relative humidity is already high at the cold exposure in both cases.

Humidity is connected to inflammatory response system. It was reported that the increase in relative humidity caused the deterioration of rheumatic arthritics [13]. For cardiovascular diseases, the effects of relative humidity were observed and, the role of inflammatory response system was argued in connection with humidity [6]. Therefore, we also checked the change of relative humidity in all of these extracted cooling and rewarming random walks. The results were shown in Table 1.

Table 1: The change of relative humidity after cold exposure.

\begin{tabular}{|c|c|c|c|}
\hline Date & $\begin{array}{l}\text { Humidity after } \\
\text { cold exposure }\end{array}$ & Date & $\begin{array}{l}\text { Humidity after cold } \\
\text { exposure }\end{array}$ \\
\hline $1 / 8 / 2003$ & Up & $1 / 26 / 2004$ & Up \\
\hline $1 / 17 / 2003$ & Up & $2 / 19 / 2004$ & $\begin{array}{l}\text { High at cold exposure } \\
(80 \%)\end{array}$ \\
\hline $1 / 19 / 2003$ & Up & $1 / 4 / 2005$ & $\begin{array}{l}\text { High at cold exposure } \\
(86 \%)\end{array}$ \\
\hline $2 / 1 / 2003$ & Up & $1 / 25 / 2005$ & Almost even \\
\hline $2 / 3 / 2003$ & $\begin{array}{l}\text { Up (down just } \\
\text { before) }\end{array}$ & $2 / 4 / 2005$ & Up \\
\hline $2 / 23 / 2003$ & Up & $2 / 6 / 2005$ & $\begin{array}{l}\text { High at cold exposure } \\
(90 \%)\end{array}$ \\
\hline $12 / 24 / 2003$ & $\begin{array}{l}\text { Up only just } \\
\text { before }\end{array}$ & $2 / 25 / 2005$ & Up only just before \\
\hline $12 / 29 / 2003$ & Up & & \\
\hline
\end{tabular}

In view of Figures 1 and $\mathbf{2}$ and Table 1, we can observe the following two main patterns for cooling and rewarming random walks.

(1) After cold exposure, both temperature and humidity increased.

(2) Relative humidity was already high at cold exposure and then temperature increased.

The analysis of gene expression is valuable to estimate the effects of meteorological factors and to speculate the mechanisms connecting weather changes and the onset of disease. The microarray data of gene expression were extracted from the database GEO. The GEO accession numbers of the used data were GSE13432 and GSE70437.

The data GSE13432 were provided by Xue et al. [9] for the purpose to study cold induced molecular mechanisms of angiogenesis in relation to adipose tissue metabolism. They used, as control, gene expression data of mice exposed to $30^{\circ} \mathrm{C}$ for both 1 week and 5 weeks. We used these data as target data rather than control data because we are interested in warming process rather than cold exposure itself.

We examined the gene expression of interleukins, especially interleukin 6 (IL-6) family and compared data for 1 week and 5 weeks. The difference of IL- 6 itself was not found, but the changes of interleukin 6 signal transducer (IL-6st) were found. The results of normalized data are shown in Table 2, where the twelve values consist of each set of three samples of mice kept at $4^{\circ} \mathrm{C}$ for 1 week, at $4^{\circ} \mathrm{C}$ for 5 weeks, at $30^{\circ} \mathrm{C}$ for 1 week, at $30^{\circ} \mathrm{C}$ for 5 weeks.

Table 2: The changes of interleukin 6 signal transducer (IL6st) during cold and warm processes. 


\begin{tabular}{|l|l|l|l|l|l|l|}
\hline Temperature & \multicolumn{2}{|l|}{ 1 week } & \multicolumn{2}{l|}{$\mathbf{5}$ weeks } \\
\hline $4^{\circ} \mathrm{C}$ & 6.73833 & 6.41872 & 7.58906 & 4.90227 & 5.69017 & 5.2098 \\
\hline $30^{\circ} \mathrm{C}$ & 5.01602 & 3.27012 & 4.7641 & 7.59789 & 7.54897 & 7.38905 \\
\hline
\end{tabular}

We compared the data $A=\{5.01602,3.27012,4.7641\}$ of mice kept at $30^{\circ} \mathrm{C}$ for 1 week and $B=\{7.59789,7.54897,7.38905\}$ of mice kept at $30^{\circ} \mathrm{C}$ for 5 weeks. The increase from $A$ to $B$ was observed, and its statistical confidence was checked by t-test with p-value 0.0271.

The GEO data of the accession number GSE70437 were supplied to study the early adaptation of brown adipose tissue to cold exposure [10]. Mice were exposed to $4^{\circ} \mathrm{C}$ for three days and control mice were kept at $22^{\circ} \mathrm{C}$. They examined increase in expression of several brown adipocyte genes related to thermogenesis as well as the gene encoding the hormone irisin. Apart from their original purpose, we examined the change of gene expression of interleukin families (Table 3).

Table 3: The changes of gene expression of interleukin 17 family.

\begin{tabular}{|c|c|c|c|c|c|c|c|c|c|c|}
\hline \multirow{2}{*}{$\begin{array}{l}\text { DNA } \\
\text { IL-17ra }\end{array}$} & \multicolumn{5}{|c|}{$22^{\circ} \mathrm{C}$} & \multicolumn{5}{|c|}{$4^{\circ} \mathrm{C}$} \\
\hline & 12 & 25 & 14 & 17 & 17 & 46 & 43 & 60 & 47 & 29 \\
\hline IL-17rd & 20 & 22 & 29 & 36 & 18 & 23 & 34 & 38 & 34 & 27 \\
\hline
\end{tabular}

We observed the increase in gene expression of the interleukin 6 signal transducer IL6st like the result of the data GSE13432. We also found the increase in gene expression of interleukin 17 family, IL-17ra, IL-17rd. IL-17 is an important interleukin for inflammation response, especially in the deterioration of rheumatic arthritis. IL-17 is closely related to IL-6 and makes a positive feedback network with IL-6. The stochastic significance of the difference between the groups of $22^{\circ} \mathrm{C}$ and $4^{\circ} \mathrm{C}$ was checked by T-test. This finding suggests a new role of IL-17 in stroke.

\section{Discussion}

Cold exposure is the most common trigger of stroke. Since there is a lag between cold exposure and the onset of stroke, to investigate the process after cold exposure is of importance.

The process after cold exposure has two aspects. One is the change of meteorological factors or weather, and the other is the change of metabolic process.

We first described the change of meteorological factors as random walks in the plane of two variables, mean temperature and relative humidity, $H(t))$,

$P(t-6)=(T(t-6), H(t-6)), P(t-5)=(T(t-5), H(t-5)), \ldots, P(t)=(T(t)$,

Where, $t$ is the day of the onset of stroke and the point $(T(t-k)$, $\mathrm{H}(\mathrm{t}-\mathrm{k})$ ) is the pair of mean temperature and relative humidity at $k$-days earlier before the day $t$. The cold exposure is supposed to happen at some day, $t-k$, before $t$. Therefore this random walk of one week contains cold exposure and ends with the onset of stroke. We explored all the cooling and rewarming random walks, $P(t-6), \ldots, P(t)$, during winter seasons from 2003 to 2005 year.

Most of the cooling and rewarming random walks had a pattern like Figure 1. There are some exceptional pattern like Figure 2. The importance of the pattern of cooling and rewarming process was pointed out by Morimoto $[3,4]$. Our findings can be considered as clear and visual description of this cooling and rewarming feature.

Given these considerations, the following warning criterion is worthy to be proposed to prevent the onset of stroke or cerebral infarction. When one encounters cold exposure or cold shock, one should check the temperature and humidity after cold shock. If one of the following two conditions is observed, the warning for the onset of stroke should be executed:

(1) After cold exposure, the both of temperature and relative humidity are likely to increase.

(2) The humidity is already high at cold exposure event, and then the temperature is likely to increase.

It is noteworthy that in Figure $\mathbf{1}$ and Table 1, the increase of relative humidity was also observed during rewarming procedure after cold exposure. In Figure 2 and Table 1, the relative humidity was already high at the day of cold exposure.

Table 4: The changes of Sirt1 during cold and warm processes.

\begin{tabular}{|l|l|l|l|l|l|}
\hline Temperature & \multicolumn{3}{|c|}{ 1 week weeks } & \multicolumn{2}{c|}{} \\
\hline $4^{\circ} \mathrm{C}$ & 8.61547 & 8.47946 & 8.12056 & 9.0859 & 9.07062 \\
\hline $22^{\circ} \mathrm{C}$ & 9.36357 & 9.4388 & 9.50956 & 9.16394 & 9.01923 \\
\hline
\end{tabular}


The combination of decreased barometric pressure and increased temperature was found to lead to a high risk of heart disease in winter seasons [4]. An increase in relative humidity was observed at the same time. A dew point association was observed for coronary atherosclerosis and congestive heart failure [14]. Here, a high dew point temperature usually means high relative humidity. Relative humidity resulted in a high inflammatory index, such as CRC values [15]. An increase in dew point temperature was associated with an increase in risk of hospitalization for coronary atherosclerosis and for congestive heart failure [14]. Relative humidity is related to a high inflammatory index, such as CRC values [15]. Relative humidity plays an important role in connection with inflammatory response system in human health.

In molecular levels, cytokines play an important role in mediating the inflammatory response. Interleukins are a subset of cytokines, and the function of the immune system depends in a large part on interleukins. Interleukins are predominately produced by and involved in the upregulation of inflammatory reactions [16]. Cytokines compose a complex network, called cytokine network, and such a network was suggested as bridge connecting the weather and the diseases $[8,17]$. Therefore, the investigation of the change of interleukins is of importance.

We explored the database of gene expression data obtained by microarrays, GEO (gene expression omnibus) which is maintained by The National Center for Biotechnology Information (NCBI). We selected gene expression data related to cooling and rewarming random walks, and found the data with GEO accession number GSE13432 (supplied by Xue et al. [9]) and GSE70437 (supplied by Marcher et al. [10]). As a cooling process, we found the increase in gene expression level of IL-6st (signal transducer of interleukin 6) in the results of GSE70437 (see in the result section). As a warming process, we noticed the increase of IL-6st in the results of GSE13432 (see in the result section). We could not find the changes of IL- 8 in these data, but the increase of IL- 8 gene expression was observed in the cooling and rewarming process [18].

It is noteworthy that the increase of receptors of interleukin 17 (IL-17) was found in the data of GSE70437 (see in the result section). IL-17 is known as an important interleukin in the inflammatory metabolism in rheumatoid arthritis. IL-17 stimulates the expression of IL- 6 and vice versa and forms a cascade of positive feedback loop with IL-6 [19]. If the loop is once driven then deterioration of rheumatoid arthritis is induced. Therefore, the finding of the increase in gene expression of IL-17 family in cooling process will provide a new aspect to roles of cytokine network in prevention of the onset of stroke.

Besides interleukins, cold shock induces variability of heat shock proteins. For example, the heat shock protein HSP70 is known to repair the protein unfolding and to modulate immune system. In recovery period at $37^{\circ} \mathrm{C}$, the induction of HSP70 is observed [18]. This process corresponds to cooling and rewarming random walks. For this induction process, it is noteworthy that another intracellular regulatory protein Sirtuin 1 (encoded by Sirt-1) plays an important role. Sirtuin-1 combines directly with heat shock transcription factor HSF1 and increases the levels of HSP70.

To search the change of gene expression of Sirtuin 1, we examined again the database GSE13432 (supplied by Xue). The result is shown in Table 4, where the reduction of Sirt-1 is observed at $4^{\circ} \mathrm{C}$ ( 1 week) compared with that at $30^{\circ} \mathrm{C}$ ( 1 week). This result was certified by stochastic confidence of $p$-value 0.007 .

The effect of cold exposure to health is modulated by both weather and the levels of metabolic states. Metabolic state declines by interleukins such as IL- 6 and TNF-alpha. On the other hand, the increase of adiponectin and Sirutin-1 improves metabolic state and leads to avoid stroke [20,21]. Further studies on the effects of effective dietary therapeutic manipulation will be expected along the research of the effects by weather

\section{Conclusion}

We described the variability of meteorological factors (mean temperature and relative humidity) as paths of random walks in the plane. We extracted random walks that correspond to paths after cold exposure to the onset of stroke, and classified the characteristic feature of cooling and rewarming random walks. These random walks were grouped into the following two cases:

(1) After cold exposure, the both of temperature and relative humidity are to increase.

(2) The humidity is already high at cold exposure event, and then the temperature is to increase.

This classification will enable us to make a forecast of stroke incidence and will supply a useful possibility to make a public warning system according to these two patterns of observation. To discuss a possible mechanism after cold exposure, we examined the gene expression data among the database GEO and found the increase of cytokine-related genes, especially interleukin 6 signal transducer IL6st and interleukin receptors of IL17. Both interleukins play an important role in inflammatory responses. These findings on gene expression corresponding to random walks will provide a new scheme that connects weather and disease, and will provide new approaches for research in the prevention of stroke.

\section{References}

1. The Eurowinter Group (1997) Cold exposure and winter mortality from ischaemic heart disease, cerebrovascular disease, respiratory disease and all causes in warm and cold regions of Europe. Lancet 349: 1341-1346.

2. Sheth T, Nair C, Muller J, Yusuf S (1999) Increased winter mortality from acute myocardial infarction and stroke: The effect of age. J Am Coll Cardiol 33: 1916-1919.

3. Morimoto $\mathrm{H}$ (2015) Patterns in stroke occurrence on warm days in winter by associations analysis. Open J Appl Sci 5: 776-782.

4. Morimoto $\mathrm{H}$ (2016) Association analysis identifies risk of ischemic heart disease when temperature increases. Int J Soc Sci Stud 4: 55-62. 
5. Sonna LA, Fujita J, Gaffin SL, Lilly CM (2002) Molecular biology of thermoregulation invited review: Effects of heat and cold stress on mammalian gene expression. J Appl Physiol 92: 1725-1742.

6. Morimoto H (2017) Trend analysis of cardiovascular events associated with meteorological factors by Bayes analysis. Gen Med Open 1: 1-4.

7. Morimoto H (2017) Bayesian analysis links weather, cerebral infarction and gene expression. Int J Collab Res Intern Med Public Health 9: 623-632.

8. Morimoto H (2017) Cytokine networks link weather and diseases. Proceedings of World Congress on Nursing \& Nurse Education, World Congress at Rome, Italy.

9. Xue Y, Petrovic N, Cao R, Larsson O, Lim S, et al. (2009) Hypoxiaindependent angiogenesis in adipose tissues during cold acclimation. Cell Metab 9: 99-109.

10. Marcher AB, Loft A, Nielsen R, Vihervaara T, Madsen JGS, et al. (2003) RNA-seq and mass-spectrometry-based lipidomics reveal extensive changes of glycerolipid pathways in brown adipose tissue in response to cold. Cell Rep 13: 2000-2013.

11. Berg HC (1983) Random walks in biology. Princeton University Press, p: 152.

12. Marsh LM, Jones RE (1988) The form and consequences of random walk movement models. J Theor Biol 133: 113-131.

13. Hollander JP, Yeostros SY (1963) The effect of simultaneous variations of humidity and barometric pressure on arthritis. AIBS Bull 13: 24-28.
14. Koken PJM, Piver WT, Ye F, Elixhauser A, Olsen LM, et al. (2003) Temperature, air pollution and hospitalization for cardiovascular diseases among elderly people in Denver. Environ Health Perspect 111: 1312-1317.

15. Ruckerl R, Ibald-Mulli A, Koenig W, Schneider A, Woelke G, et al. (2006) Air Pollution and Markers of inflammation and coagulation in patients with coronary heart disease. Am J Respir Crit Care Med 173: 432-441.

16. Kostulas N, Kivisäkk P, Huang Y, Matusevicius D, Kostulas V, et al. (1998) Ischemic stroke is associated with a systemic increase of blood mononuclear cells expressing interleukin-8 mRNA. Stroke 29: 462-466.

17. Morimoto H (2017) Trend analysis of cardiovascular events associated with meteorological factors by Bayes analysis. Gen Med Open 1: 1-4.

18. Gon Y, Hashimoto S, Matsumoto K, Nakayama T, Takeshita I, et al. (1998) Cooling and rewarming-induced IL-8 expression in human bronchial epithelial cells through p38 MAP kinase-dependent pathway. Biochem Biophys Res Commun 249: 156-160.

19. Lubberts E (2008) IL-17/Th17 targeting: On the road to prevent chronic destructive arthritis? Cytokine 41: 84-91.

20. Martins IJ (2016) Heat shock gene Sirtuin 1 regulates post-prandial lipid metabolism with relevance to nutrition and appetite regulation in diabetes. Int J Diab Clin Diagn 3: 20.

21. Martins IJ (2016) Diet and nutrition reverse type 3 diabetes and accelerated aging linked to global chronic diseases. J Diab Res Ther 2: 2. 